\title{
Um Caso Prático de Implantação da Gerência de Risco em Ambientes de Desenvolvimento Distribuído de Software, baseado no Modelo CMMI
}

\author{
Rafael Prikladnicki ${ }^{1}$, Geraldo Gomes, Azriel Majdenbaum, Marcelo Hideki \\ Yamaguti $^{1}$, Dante Antunes, Silvia Oliveira ${ }^{1}$, Jorge Luis Nicolas Audy ${ }^{1}$ \\ ${ }^{1}$ Pontifícia Universidade Católica do Rio Grande do Sul - PUCRS - FACIN \\ Av. Ipiranga, 6681 - Prédio 16 - Sala 106 - Porto Alegre, RS - CEP 90619-900 \\ \{rafael, yamaguti, soliveira, audy\}@inf.pucrs.br
}

\begin{abstract}
The purpose of this paper is to present the experience of implementing a risk management process in a distributed software development environment, considering the CMMI level 3 standard. The experience is described and some lessons learned are identified and presented.
\end{abstract}

Resumo. $O$ objetivo deste artigo é apresentar um relato de implantação de um processo de gerência de risco em um ambiente de desenvolvimento distribuído de software, visando atender os requisitos do modelo CMMI nível 3. A implantação foi realizada em um centro de desenvolvimento de software de uma organização multinacional. Ao final, identificam-se algumas lições aprendidas do acompanhamento realizado.

\section{Introdução}

$\mathrm{Na}$ área de software, a busca pela sobrevivência em um mercado cada vez mais globalizado tem tornado a qualidade dos produtos uma necessidade evidente. Os projetos de desenvolvimento de software têm tido inúmeros problemas relacionados à sua gerência, tais como gastos além do orçado, atrasos na entrega e não atendimento às necessidades dos clientes. Além disso, com o objetivo de obter custos menores, maior qualidade no processo de desenvolvimento e a possibilidade de ter recursos em âmbito global, muitas organizações começaram a investir em ambientes de desenvolvimento distribuído de software (DDS) [1]. Por este motivo, o gerenciamento de projetos de software tem se tornado um desafio constante, especialmente quando envolvem novas tecnologias [2] e novos ambientes. $\mathrm{O}$ fracasso nos projetos muitas vezes é resultado de falhas na identificação e gerenciamento de múltiplos riscos inerentes ao ambiente no qual o projeto está inserido [3].

Soma-se a isso o fato de que, além de ter-se como necessidade a melhoria da qualidade do produto final, muitas empresas têm se preocupado em melhorar o próprio processo de desenvolvimento como forma de garantir a qualidade do produto em si. Neste contexto, surgem modelos de qualidade [4], como, por exemplo, o Capability Maturity Model (CMM), usados pelas organizações como guia para definir seus processos de software e a maturidade dos mesmos, tanto como para orientar um trabalho de melhoria destes processos. Uma das áreas abordadas por este modelo diz respeito à gerência dos riscos envolvidos em projetos de desenvolvimento de software. 
O objetivo deste artigo é apresentar um relato da implantação de um processo de gerência de risco em um ambiente de DDS, visando atender os requisitos do modelo CMMI nível 3. Este estudo pode ser caracterizado como exploratório, baseando-se em um estudo de caso desenvolvido em um centro de desenvolvimento de software de uma empresa multinacional, reconhecido como SW-CMM nível 2 desde janeiro de 2003.

O artigo está organizado em 4 seções. A seção 2 apresenta o referencial teórico envolvido. Na seção 3 apresenta-se o relato da experiência de implantação do processo. $\mathrm{Na}$ seção 4 apresentam-se as lições aprendidas. A seção 5 apresenta as considerações finais. As referências bibliográficas são apresentadas na seção 6.

\section{Referencial Teórico}

\subsection{Gerência de Risco}

Risco como ciência nasceu no século XVII, no Renascimento. Foi em uma tentativa de entender os jogos de azar que Blaise Pascal, em 1654, descobriu a "teoria da probabilidade" e criou o "triângulo de Pascal", que determina a probabilidade de ocorrer possíveis saídas, dado certo número de tentativas [5]. Na área de software, o risco foi representado de forma sistemática por Barry Boehm, nos anos 80, através do modelo em espiral, que tem como princípio ser iterativo e dirigido a análise do risco em cada iteração [6]. A palavra "risco" deriva do italiano antigo "risicare", que significa "ousar" [5]. Nesse sentido, risco é uma opção e não um destino. Por isso, requer mais do que processos competentes e uma habilidade de pensar intuitivamente; requer disciplina. A esta disciplina dá-se o nome de 'gerência de risco'.

Atualmente, a gerência de risco na área da Engenharia de Software é uma evolução do conceito de risco, que passou de uma análise dentro do modelo de desenvolvimento, para uma gerência, que deve permear todos os processos do ciclo de vida do software. O gerenciamento de risco é um processo pró-ativo, que é invocado para tentar eliminar problemas potenciais e as incertezas antes que eles ocorram e, com isso, aumentar a probabilidade de sucesso do projeto [6].

\subsection{Desenvolvimento Distribuído de Software (DDS)}

Observou-se na última década um grande investimento na conversão de mercados nacionais em mercados globais, criando novas formas de competição e colaboração [1]. Uma crescente demanda, atingida pela escassez de recursos capacitados, custos mais baixos e a pressão para o time-to-market e para o desenvolvimento round-the-clock (desenvolvimento quase que contínuo) fez com que muitas organizações encontrassem uma alternativa no DDS [7, 8].

O DDS tem sido caracterizado principalmente pela colaboração e cooperação entre departamentos de organizações e pela criação de grupos de desenvolvedores que trabalham em conjunto, mas estão localizados em cidades ou países diferentes. Segundo Carmel [1], o DDS está causando um grande impacto não apenas no mercado propriamente dito, mas na maneira como os produtos de software estão sendo criados, modelados, construídos, testados e entregues para os clientes. Além disso, Carmel [1] destaca que trabalhar com DDS é um dos maiores desafios que o atual ambiente de negócios apresenta do ponto de vista do processo de desenvolvimento de software.

Neste sentido, o DDS tem atraído um grande número de pesquisas na área de Engenharia de Software ([7, 8, 9]). Os engenheiros de software têm reconhecido a 
influência desta nova forma de trabalho no seu dia-a-dia e estão em busca de modelos que facilitem o desenvolvimento de software com equipes geograficamente distantes..

\subsection{Gerência de Risco em Projetos de DDS}

No DDS, a gerência de risco é tida como uma área sensível e com uma grande importância. Um estudo feito por [10] levantou que uma das principais diferenças entre desenvolver software de forma distribuída e de forma centralizada está na gerência dos riscos. Além disso, este estudo também identificou que a gerência efetiva dos riscos foi uma das formas de solucionar os problemas existentes nos projetos distribuídos. Por este motivo, a gerência de risco tem uma grande importância em projetos com equipes distribuídas, sendo estas equipes da mesma empresa ou de empresas diferentes.

Segundo Karolak [8], gerenciar riscos faz parte de qualquer projeto, e os riscos em projetos de DDS tendem a estar mais centrados em aspectos não tão visíveis. Ainda de acordo com este autor, podem existir três categorias de riscos em projetos de DDS, e riscos presentes em mais de uma categoria devem estar no topo da lista de prioridades:

- Organizacional: envolve os papéis e responsabilidades dos integrantes das equipes dos projetos (tarefas e limitações associadas). Um exemplo seria o não entendimento de quem pode tomar certas decisões;

- Técnico: envolve métodos e ferramentas para solucionar problemas técnicos, tais como melhorar o desempenho do produto, desenvolvimento, etc. Riscos incluem o mau uso da metodologia de desenvolvimento, arquitetura inapropriada e impossibilidade de integração entre módulos;

- Comunicação: envolve a infra-estrutura que os integrantes das equipes utilizam para se comunicar. Riscos incluem discussões mal interpretadas, idéias mal formuladas, comunicação inadequada.

Segundo Prikladnicki [10], a gerência de riscos em projetos de DDS deve ocorrer tanto em nível de projeto e principalmente em um nível organizacional, no sentido de analisar as vantagens de um determinado projeto ser desenvolvido de forma distribuída e os riscos envolvidos. Além disso, o autor sugere que, uma vez que a análise de risco foi realizada e decidiu-se que um determinado projeto será desenvolvido de forma distribuída, os riscos identificados devem ser repassados ao gerente do projeto para que ele agregue aos riscos do projeto como um todo e planeje ações para lidar com os riscos identificados.

\subsection{Capability Maturity Model Integration - CMMI}

O CMMI nasceu da necessidade de melhorar o modelo SW-CMM e de compatibilizar o SW-CMM com a norma ISO 15.504, desenvolvida pelo projeto SPICE [11]. As principais mudanças existentes entre o SW-CMM e o CMMI envolvem algumas diferenças de terminologias e a estrutura [12]. A principal diferença está no fato de que o SW-CMM organiza os processos de software em estágios (5 níveis), enquanto o CMMI também o faz de uma forma que se convencionou chamar de "representação contínua" [13], também utilizada na ISO 15.504 [14]. Portanto, o CMMI admite as duas representações e a organização, ao implantar o modelo, pode decidir qual representação é mais adequada a sua realidade. Além disso, através do processo de adequação à ISO 15.504, é possível a "certificação cruzada", de modo que organizações avaliadas por um modelo tenham sua avaliação reconhecida pelo outro modelo. 
Em relação à área de processo de gerência de risco, no modelo CMMI a gerência de risco (RSKM) é representada por uma área de processo do nível 3, na categoria de processos de gerência de projeto, enquanto que no SW-CMM aparecia no nível 3 dentro da área chave de Gerência Integrada de Projeto (IPM), representada pela atividade 10. Segundo o CMMI, o objetivo da gerência de risco é identificar problemas potenciais antes que eles ocorram, para que atividades de redução de riscos possam ser planejadas ao longo do ciclo de vida, reduzindo o impacto nos objetivos dos projetos. A área de processo está definida em um objetivo genérico e três objetivos específicos, que englobam a preparação para a gerência de risco, a identificação e análise dos riscos e a redução dos riscos. Além disso, o modelo sugere como artefatos típicos a geração de listas de riscos; categorias de risco; critérios de avaliação, categorização e priorização de riscos; estratégias para planejar a gerência de risco; planos de resposta aos riscos; etc.

\section{Implantação da Gerência de Risco em Ambientes de DDS, baseado no CMMI}

Tendo como base a área de processo de gerência de risco do nível 3 do modelo CMMI, e considerando um ambiente de desenvolvimento distribuído de software, descreve-se a seguir a implantação do processo desenvolvido. O relato foi acompanhado em uma organização de desenvolvimento de software durante todas as fases do processo (incluindo o planejamento, desenvolvimento e implantação).

\subsection{Caracterização da organização}

A implantação da gerência de risco foi acompanhada em uma unidade de desenvolvimento de software de uma organização de grande porte, com sede nos Estados Unidos. A unidade onde o estudo foi aplicado está localizada em uma cidade do Estado do Rio Grande do Sul. Ela possui mais de 180 colaboradores que trabalham em projetos que atendem as necessidades da área de TI da empresa (demanda interna). A unidade possui nível dois de maturidade de desenvolvimento de software reconhecida pelo padrão SW-CMM desde 2003. O processo tem como base o MSF (Microsoft Solution Framework) para o desenvolvimento de software e a metodologia do PMI para o gerenciamento de projetos. Além disso, o RUP também é utilizado, dependendo das características dos projetos. Estes processos não são aplicados na sua totalidade, sendo adaptados à realidade da organização e desenvolvidos pelos próprios funcionários. Todos os processos são controlados pelo SEPG (Software Engineering Process Group).

\subsection{Implantação do processo de Gerência de Risco no contexto do CMMI nível 3}

A implantação do processo de gerência de risco se deu ao longo de um plano para definir e implantar parte dos processos de software definidos no CMMI nível 3. O processo iniciou no final de 2003 e está em andamento. Especificamente em relação ao processo de gerência de risco, o mesmo pode ser organizado em fases, conforme o esquema cronológico abaixo (Figura 1).

\begin{tabular}{|l|l:l|l|l|l|}
\hline Fases & & $\mathbf{2 0 0 3}$ & 2004 \\
\hline $\begin{array}{l}\text { Desenvolvimento } \\
\text { Capacitação }\end{array}$ & Outubro & Novembro & Dezembro & Janeiro & Fevereiro \\
Implantação & & & & & \\
\hline
\end{tabular}

Figura 1 - Fases da implantação do processo de Gerência de Risco 
Fase 1 - Desenvolvimento: Na primeira fase, foi desenvolvido um processo de gerência de risco de acordo com a realidade da organização. Na época, a organização tinha recentemente alterado a sua estratégia no que diz respeito ao projeto de melhoria de processo de software. Inicialmente, em março de 2003, a empresa havia realizado um planejamento para ser reconhecida como uma organização nível 3 no modelo SWCMM. Mas com a avaliação positiva da subsidiária na Índia como sendo uma organização nível 2, em setembro alterou-se a estratégia, onde os esforços deveriam ser concentrados no reconhecimento no modelo CMMI, e devia ser um trabalho conjunto, entre as unidades do Brasil e da Índia. Naquele momento, a interação de ambas as unidades era apenas com a matriz, nos Estados Unidos. A vantagem desta abordagem seria o desenvolvimento e implantação do mesmo processo, para no futuro haver uma interação mais fácil entre as unidades nos projetos de desenvolvimento de software, tornando o DDS uma realidade ainda mais presente na organização. O planejamento e as vantagens e desvantagens desta estratégia não serão discutidos, pois o grupo vivenciou apenas este processo e não tem como fazer uma avaliação mais abrangente. Um estudo completo sobre a definição de processos globais pode ser encontrado em Vanzin [15].

Sendo assim, definiu-se um projeto interno a ser conduzido por um grupo de trabalho distribuído, com quatro integrantes no Brasil e quatro na Índia, sendo que em ambos os locais haveria um representante de cada SEPG. Além disso, a equipe no Brasil, por já possuir um processo de gerência de risco definido, iria atuar como equipe coordenadora e responsável pela gerência de configuração dos artefatos. No início da interação, fotos dos integrantes da equipe foram trocadas, objetivando a integrar e facilitar a interação. Houve uma primeira reunião onde a equipe do Brasil apresentou o processo e os artefatos existentes (descrição do processo, ferramenta desenvolvida, lista de riscos genéricos, plano de riscos e dicas de utilização do processo). A proposta de processo desenvolvida no Brasil, que pode ser encontrada em Prikladnicki [16], foi então enviada para revisão pela equipe na Índia. Esta revisão identificou alguns pontos fracos e ambas as organizações sugeriram mudanças. Estas mudanças foram adicionadas ao processo e novas revisões foram feitas. Reuniões à distância foram realizadas para analisar o processo e os artefatos criados. Ao final, o processo foi criado, com pequenas alterações em relação ao processo proposto, devido a alguns ajustes necessários para refletir também a realidade da Índia.

Fase 2 - Capacitação: Após a realização dos pequenos ajustes para adequar o processo à realidade da organização, a equipe no Brasil ficou responsável pela criação do material de treinamento. Este material seguiu alguns procedimentos padrão definidos na organização e foi gerado com o objetivo de treinar todos os gerentes de projetos e opcionalmente gerentes de desenvolvimento e líderes técnicos.

Fase 3 - Implantação: Com o processo criado e as equipes treinadas, foi possível definir alguns projetos pilotos para começarem a utilizá-lo. Os projetos selecionados eram projetos distribuídos, mas não envolviam interações com equipes de desenvolvimento na Índia, apenas com equipes localizadas nos EUA. Por este motivo, o grupo de pesquisa vivenciou apenas a utilização do processo nos projetos pilotos desenvolvidos no Brasil. Sendo assim, definiram-se três projetos pilotos, onde haveria um integrante do grupo de trabalho atuando como mentor e suporte para qualquer dificuldade. Os projetos pilotos foram avaliados, melhorias foram propostas e então os novos projetos na organização começaram a utilizar o processo de gerência de risco desenvolvido. 


\section{Lições Aprendidas}

Com a implantação do processo de gerência de risco em três projetos pilotos, foi possível vivenciar o planejamento, o desenvolvimento e a implantação do processo. Além disso, identificaram-se algumas lições aprendidas, que são apresentadas a seguir.

Lição 1: O desenvolvimento de um processo deve ser encarado como um projeto.

Ao contrário do que ocorreu no projeto do nível 2 do modelo SW-CMM, a organização percebeu que era necessário o planejamento das atividades para dar visibilidade do projeto como um todo, além de ter o comprometimento do grupo de trabalho. Por este motivo, a criação de um grupo de trabalho e de um plano de projeto, a definição dos objetivos, artefatos de entrega e um relatório de acompanhamento foi muito importante. Além, disso, o fato de ter sido definido como um projeto interno foi importante de modo a facilitar o acompanhamento do projeto de melhoria contínua como um todo.

Lição 2: $O$ desenvolvimento de um processo deve ser de responsabilidade da organização como um todo

O grupo de trabalho que participou da adequação do processo trabalhava em diferentes equipes dentro da organização. Por este motivo, obteve-se uma representatividade considerável da organização como um todo. Além disso, o processo foi concebido tendo como requisitos as necessidades da própria organização, utilizando uma estratégia bottom-up, onde a criação e a implantação do processo ocorreram a partir dos níveis hierárquicos inferiores, tornando mais fácil a sua implantação.

Lição 3: A integração com equipes mais experientes motivou e aumentou o conhecimento do grupo de trabalho

Apesar da necessidade de realizar pequenas mudanças no processo devido a realidade da Índia, a grande interação com o grupo de trabalho localizado naquele país foi bastante positiva no sentido de enriquecer o processo com uma outra visão, de pessoas mais experientes no modelo CMMI, que era novo para a equipe no Brasil.

Lição 4: O processo de gerência de risco deve levar em conta o conhecimento organizacional, principalmente quando se atua em projetos distribuídos

Verificou-se que muitos projetos possuíam características parecidas e, portanto, alguns riscos poderiam ser iguais. Neste sentido, foi proposta a criação de um repositório com informações de riscos em projetos anteriores, bem como características destes projetos.

Lição 5: $O$ processo de gerência de risco deve incentivar a pró-atividade e a comunicação constante

Verificou-se que a gerência de risco aumentou consideravelmente a necessidade de comunicação entre os interessados. Ao mesmo tempo, este aumento de comunicação trouxe benefícios no sentido de haver um maior controle do projeto e dos riscos envolvidos. No caso de projetos distribuídos, uma preocupação adicional foi criada, envolvendo um melhor planejamento de toda a infra-estrutura de comunicação.

Lição 6: O processo de gerência de risco deve ser suportado por uma ferramenta Uma ferramenta foi desenvolvida para facilitar o uso do processo na organização. Percebeu-se que a existência da ferramenta guiou o aprendizado do processo e auxiliou principalmente na extração de diversos dados referentes aos riscos do projeto, além de facilitar a criação e controle de relatórios gerenciais periódicos.

Lição 7: Todos os riscos identificados devem ser documentados para refletir nos próximos projetos

Todos os riscos identificados eram monitorados e controlados e ao final do projeto eram documentados de forma a serem consultados nos próximos projetos. Isto foi útil para 
manter uma uniformidade nos riscos identificados e na atualização constante de uma lista genérica de riscos.

\section{Lição 8: A organização deve estar preparada para a implantação de um novo processo e deve existir uma equipe de suporte à implantação deste}

Uma das maiores dificuldades na implantação do processo foi a mudança de cultura organizacional. Pelo fato de a organização nunca ter possuído um processo para gerenciar efetivamente os riscos, havia uma idéia inicial de que o processo estava sendo executado apenas por que era necessário e estava descrito no modelo CMMI. Com o tempo, à medida que os resultados positivos foram surgindo, as equipes começaram a perceber que o processo trazia benefícios aos projetos. Além disso, após o treinamento, identificaram-se algumas dificuldades na implantação do processo, principalmente no que diz respeito à utilização da ferramenta de apoio. Por isto, o grupo de trabalho auxiliou as equipes de projeto, facilitando o entendimento e aplicação do novo processo.

\section{Considerações finais}

A natureza dos projetos de software (dinâmicos e únicos) cria riscos que deveriam ser gerenciados pela empresa como um todo e em especial pela área de TI. A fim de obter sucesso nos projetos de software as organizações precisam gerenciar os vários riscos envolvidos. A qualidade dos produtos pode depender da forma com que os riscos são gerenciados ao longo do processo. Mas apesar das evidências de que a gerência de risco pode ajudar no alcance do sucesso nos projetos de software, essa área ainda encontra obstáculos para ser institucionalizada pelas empresas [16].

Uma avaliação de melhoria de processos conduzida em 1999 na Austrália constatou que a gerência de risco é executada de forma planejada e com acompanhamento em somente $12 \%$ das empresas daquele país; $48 \%$ a executam de maneira informal e $40 \%$ não executam o processo [17]. No Brasil, a pesquisa da Secretaria de Planejamento em Informática (SEPIN) em 2001 demonstrou que somente $11,43 \%$ das 446 empresas pesquisadas executam o processo de gerência de risco [18]. Desta forma, este estudo buscou compartilhar a experiência de desenvolver e implantar a gerência de risco em uma organização em um contexto de DDS.

A análise do relato de experiência descrito indica que um dos principais motivos para a utilização ineficiente ou não utilização da gerência de riscos nas organizações que desenvolvem software pode ser atribuído à falta de documentação de experiências de sucesso ou de fracasso. Além do conhecimento sobre gerência de riscos, a análise de experiências passadas é fundamental para auxiliar os gerentes de projeto no planejamento e controle de riscos. O processo de gerenciamento de risco formal é recomendado para gerenciar problemas complexos associados com projetos de desenvolvimento de software [17]. Porém poucas organizações tem tido sucesso ao utilizar as abordagens propostas por diversos autores $[2,3,16]$. Isto ocorre porque o efetivo sucesso da gerência de risco está intimamente ligado à mudança de mentalidade organizacional e individual. Implantar a gerência de risco nas organizações para diminuir os insucessos é um desafio para profissionais da área de desenvolvimento de software. Da mesma forma, existe uma série de problemas e desafios inerentes ao desenvolvimento de software. E o DDS acentuou alguns dos desafios existentes, ao acrescentar fatores como dispersão geográfica e dispersão temporal e acrescentou novos desafios ao processo de desenvolvimento. Por isso, a gerência efetiva dos riscos não pode ser jamais desprezada. 


\section{Referências bibliográficas}

[1] Herbsleb, J. D. and Moitra, D. (2001). Guest editors' introduction: Global software development. IEEE Software, 18(2):16-20.

[2] Schwalbe, K. (2000). Information Technology Project Management. Course Technology, Cambrigde.

[3] Roy, G. G.; Woodings, T. L. (2000). A Framework for Risk Analysis in Software Engineering, In: Proceedings of the $7^{\text {th }}$ Asia-Pacific Software Engineering Conference.

[4] Oliveira, K.; Rocha, A.; Weber, K. (2002). Workshop on Software Quality. In: Proceedings of the International Conference on Software Engineering. Flórida, EUA.

[5] Bernstein, P. (1997). Desafio aos deuses: a fascinante história do risco. Campus.

[6] Boehm, B. (1991). Software risk management: principles and practices. Piscataway: IEEE Software, v. 8, p. 32-41.

[7] Carmel, E. (1999). Global Software Teams - Collaborating Across Borders and Time-Zones. Prentice Hall, EUA.

[8] Karolak, D. W. (1998). Global Software Development - Managing Virtual Teams and Environments. Los Alamitos, IEEE Computer Society, EUA.

[9] Kiel, L. (2003). Experiences in Distributed Development: A Case Study. Proceedings of the International Workshop on Global Software Development at ICSE.

[10] Prikladnicki, R. (2003). MuNDDoS: Um Modelo de Referência para Desenvolvimento Distribuído de Software. Dissertação de Mestrado. PPGCC - PUCRS.

[11] Côrtes, M. (2001). Modelos de Qualidade de Software, Campinas, UNICAMP.

[12] ISO/IEC 15504 (1998). Software Process Assessment, Technical Report.

[13] CMU/SEI-2002-TR-011 - Capability Maturity Model Integration (CMMI), version 1.1, Continuous Representation.

[14] CMU/SEI-2002-TR-012 - Capability Maturity Model Integration (CMMI), version 1.1, Staged Representation.

[15] Vanzin, M., Blois, M, Prikladnicki, R., Ceccato, I., Antunes, D. (2005). Global Software Processes Definition in a Distributed Environment. In Proceedings of the 29th Annual NASA/IEEE Software Engineering Workshop.

[16] Prikladnicki, R., Yamaguti, M. H., Antunes, D. C. "Risk Management in Distributed Software Development: A Process Integration Proposal", In: PRO-VE 2004, $5^{\text {th }}$ IFIP Working Conference on Virtual Enterprises at $18^{\text {th }}$ IFIP World Computer Congress

[17] Kwak, Y. H.; Stoddard, J. (2003). Project Risk Management: lessons learned from software development. Technovation, In Press, Corrected.

[18] Rout, T.; Tuffley, A. (2000). The rapid assessment of software process capability. In: Spice International Conference on SPI and Capability Determination, Ireland.

[19] Ministério da Ciência e Tecnologia - SEPIN. (2002). Relatório preliminar da qualidade e produtividade de software. Brasília. Disponível em: www.mct.org.br.

\footnotetext{
i Estudo realizado pelo Grupo de Pesquisa em Desenvolvimento Distribuído de Software do PDTI, financiado pela Dell Computadores do Brasil Ltda. com recursos da Lei 8.248/91.
} 